\title{
Emotional face expression profiles supported by virtual human ontology
}

\author{
By A. García-Rojas*, F. Vexo, D. Thalmann, A. Raouzaiou, K. Karpouzis, \\ S. Kollias, L. Moccozet and N. Magnenat-Thalmann
}

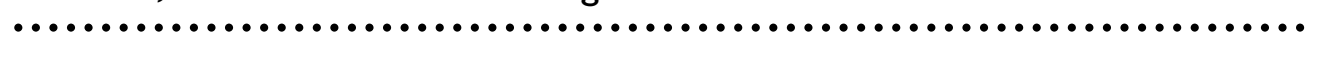

Expressive facial animation synthesis of human like characters has had many approaches with good results. MPEG-4 standard has functioned as the basis of many of those approaches. In this paper we would like to lay out the knowledge of some of those approaches inside an ontology in order to support the modeling of emotional facial animation in virtual humans (VH). Inside this ontology we will present MPEG-4 facial animation concepts and its relationship with emotion through expression profiles that utilize psychological models of emotions. The ontology allows storing, indexing and retrieving prerecorded synthetic facial animations that can express a given emotion. Also this ontology can be used a refined knowledge base in regards to the emotional facial animation creation. This ontology is made using Web Ontology Language and the results are presented as answered queries. Copyright (C) 2006 John Wiley E Sons, Ltd.

Received: 10 April 2006; Revised: 2 May 2006; Accepted: 10 May 2006

KEY WORDS: ontology; MPEG-4 facial animation; emotion expression

\section{Introduction}

The real world actions of a human can be transferred into a virtual environment through a representative (avatar), while the virtual world perceives these actions and responds through respective system avatars which may express their emotions using human-like expressions and gestures. Authors of human face animation synthesis are closely attached to the MPEG-4 standard because it provides a structured control of a character's face. This paper is based on the work of Raouzaiou et al. ${ }^{1}$ who have presented approaches to synthesize MPEG-4 facial expressions based on discrete and dimensional emotion representation models. ${ }^{2}$ The advantage of the mentioned approaches is that real and naturalistic data captured with emotion induction techniques are used and, as a result, the obtained measurements are far more realistic that those portrayed in most facial expression databases, such as MIT's or Eckman's.

*Correspondence to: A. García-Rojas, VRLab-EPFL, CH-1015 Lausanne, Switzerland. E-mail: alejandra.garciarojas@epfl.ch

Contract/grant sponsor: EC; Swiss Federal Office for Education and Science in the framework of the Education Networks of Excellence IST-HUMAINE; IST-AIM@SHAPE.
The potential and the results of the knowledge posted by those approaches are not of the common understanding, in the sense that they are described in proprietary rule-based forms, inconsistent with emerging knowledge representation concepts. Synthesis facial animation comprehends many fields of knowledge: psychology, animation control, standards, etc. and using an ontology is a good solution for a formal specification of a shared knowledge. ${ }^{3}$ In this paper we propose to present this knowledge using ontologies for a common understanding. We aim to structure concepts involved in the work done in animation of facial expressions to provide higher descriptors.

The MPEG-7 standard caters for the interchangeable description of structured information. Shape descriptors are proposed in order to support the extraction of low level features from 2D and 3D models. Zaharia et al. ${ }^{4}$ propose a MPEG-7 3D shape descriptor that computes the histogram of the shape index over the whole 3D surface. Such feature extraction can further be incorporated into a $3 \mathrm{D}$ model retrieval system. The basic approach ${ }^{5}$ usually consists in providing a target 3D shape and in retrieving from the database 3D shapes that show similarities in the features space with the target shape. An alternate application classifies similar 3D shapes into clusters. Although virtual human models involve 3D shapes for the 
body representation, they contain much higher level semantic features. Therefore, specific high-level descriptors are required in order to develop an accurate model retrieval systems. Semantics aims at easing the construction, functionality and control of VHs. For modeling the knowledge involved in the creation of virtual humans, Gutierrez et al. ${ }^{6}$ have presented an ontology-based approach. They present the complexity this work carries and a general framework.

To explain the objective of this ontology we describe two kinds of scenarios. The first one is for retrieving animation files that have been annotated with high-level descriptors, for example searching for a face animation that express a particular emotion. This is to promote the creation of reusable, scalable, and adaptive content in virtual environments. The second scenario is where the ontology can provide a kind of expertise in the knowledge domain. For example, to search for the parameter values needed to generate certain kind of expression like: feature points involved, range of their FAPs values, emotion-activation value, etc.

This paper is organized as follows: the next section presents the ontology domain, which is composed by MPEG-4 definitions and the representation of emotion. After we will present the description of face expression profiles which are definitions of previews work on facial expression synthesis. Subsequently, we present the ontology and an example of the ontology population with the results of the expression synthesis approach. Finally, the usage scenarios of the ontology and the conclusions.

\section{Ontology Domain}

The goal of the ontologies is to lay down the knowledge and express it in a way which is understandable for both humans and computers. This ontology would help to provide the right animation for a desired expression of emotion. To illustrate this we provide the following questions: What are the face animations for expressing sad emotion? What kinds of anger expression do we have? What is the range of FAP values for the following emotions: worried, terrified? Given a set of FAP values what is the emotion that can be produced?

First, we need to define the knowledge of the face animation object of MPEG-4 in order to describe the animation structure of VHs. This structure is used to establish a relationship between the animation components and emotional parameters. The following subsections explain how it is modeled within the ontology domain. The ontology is created using Ontology Web Language. ${ }^{7}$
OWL is a formal language that precisely specifies the semantic relationships among entities; it facilitates greater machine interpretability of web content than that supported by XML, RDF, and RDF Schema (RDF-S). OWL language offers more ways of entity relation, cardinality relation between entities and other logical operations when compared with older languages. The ontology design was made using Protégé ontology editor ${ }^{8}$ which is an open source knowledge-base framework.

\section{MPEG-4 Face Animation Object}

MPEG-4 has been exploited because it makes it possible to have a structured control of the character. Some of the standard advantages are: very low bit rate coding, high quality and customizable rendering, scalability, etc.

In the framework of MPEG-4 standard, some parameters have been specified for face and body animation (FBA). For facial definition, MPEG-4 specifies 84 feature points on the neutral face, ${ }^{9}$ which provides spatial reference for the facial animation parameters (FAPs) definition. The FAP set contains two high-level parameters, visemes and expressions. Facial shape is defined by the facial definition parameters (FDP) and facial deformation tables (FDT) nodes. FDP contains the feature points in the face shape and the FDT contains the deformation of the model face as a function of its animation. For animating a face, FAP node has the translations of feature points, expressed in FAP Units; they can produce high quality facial expressions and speech pronunciation. FAPs that can be used together are represented in groups in order to facilitate the animation. ${ }^{10}$ This structure of nodes is represented in the ontology in Figure 1.

\section{Emotional Expression Representation}

Depending on the context of interaction, one might prefer to choose a discrete emotion representation models, such as Eckman's, over a dimensional. For example, if the objective of an application is classification of images or videos into specific categories, it is better to use discrete representations. On the other hand, dimensional models perform better when capturing subtle emotions (everyday human discourse), and can be differentiated between the two principal axes, for example, in an anger detection application used in automated phone centers. Besides these representations, component process models, such as Stacy-Marsella's or Scherer's appraisal checks, aim at mimicking human brain processes when confronted 


\section{computer animation \& virtual worlds

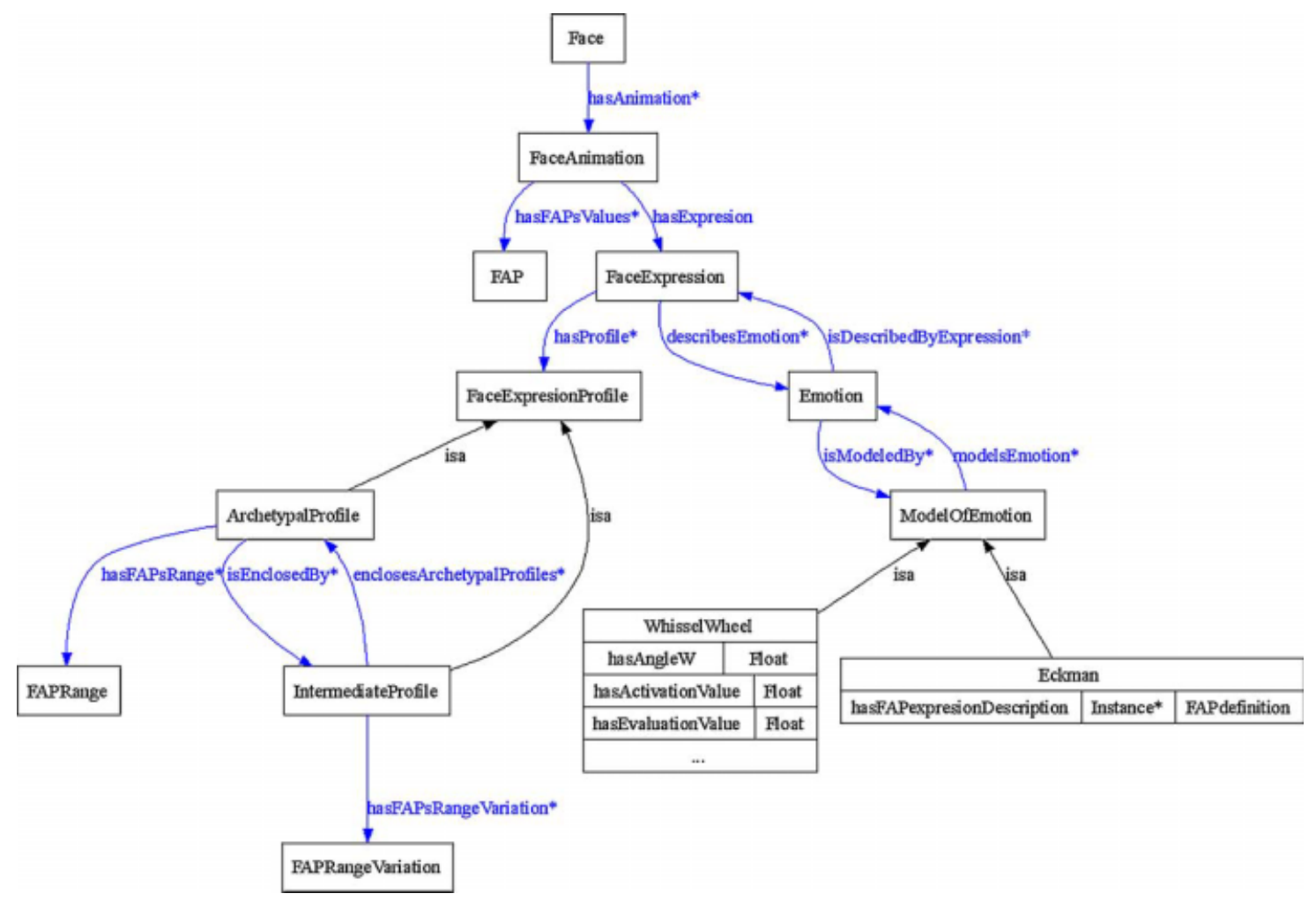

Figure 1. Representation of the facial and body animation object of MPEG-4 in the ontology.

with new events. In these situations, several aspects of a stimulus are taken into account, such as its disposition against the aims of the subject, its novelty, etc. Albeit they are useful in modeling human-human discourse, there are several unclear aspects in these models that hamper the development of related computational models, such as timing or sequencing of processes.

$\mathrm{VH}^{\prime}$ 's face is capable of having explicit synthetic expressions by itself within animations ${ }^{11,12}$ according to a given emotion. Eckman's ${ }^{13}$ model of emotion has been widely accepted to describe the six archetypal emotions. Within MPEG-4 for each of these basic emotions a list of FAPs is associated (those FAPs are animated to produce the specified emotion). However, this model is not sufficient to produce a varied range of emotions; therefore, we need to incorporate a more transparent and continuous representation of emotions. Whissel's ${ }^{14}$ activationevaluation space is a simple circular representation that captures a wide range of emotions and simplifies them in two dimensions: activation and evaluation. As shown in Figure 2, the vertical axis represents the activation value and the horizontal one the evaluation value. Universal emotions, as well as intermediate ones, are represented as points in the activation-evaluation space.
The ontology diagram of the representation of emotions is presented in the Figure 3. It shows that one emotion can be modeled by one or more models of emotion. In next section we will describe prior work on facial expression synthesis that group facial expression inside profiles. These profiles are the connection between MPEG-4 and the mentioned models of emotion.

\section{Face Expression Profiles}

As general rule, one can define six general categories for facial expressions, each categorized by an archetypal emotion. Within these categories, expressions are described by different emotional intensities, as well as minor variations in expression details. The need for different expression profiles ${ }^{1}$ arises from the fact that the same emotion may be expressed via the face using different facial deformations, subject to personal traits or different flavors in expressivity. This concept is useful in both analysis and synthesis purposes, since it allows for personalized, diverse expressions, thus reducing the possibility of robot-like faces. In the following subsections we will explain the profiles defined for archetypal and inter- 


\section{VERY ACTIVE}

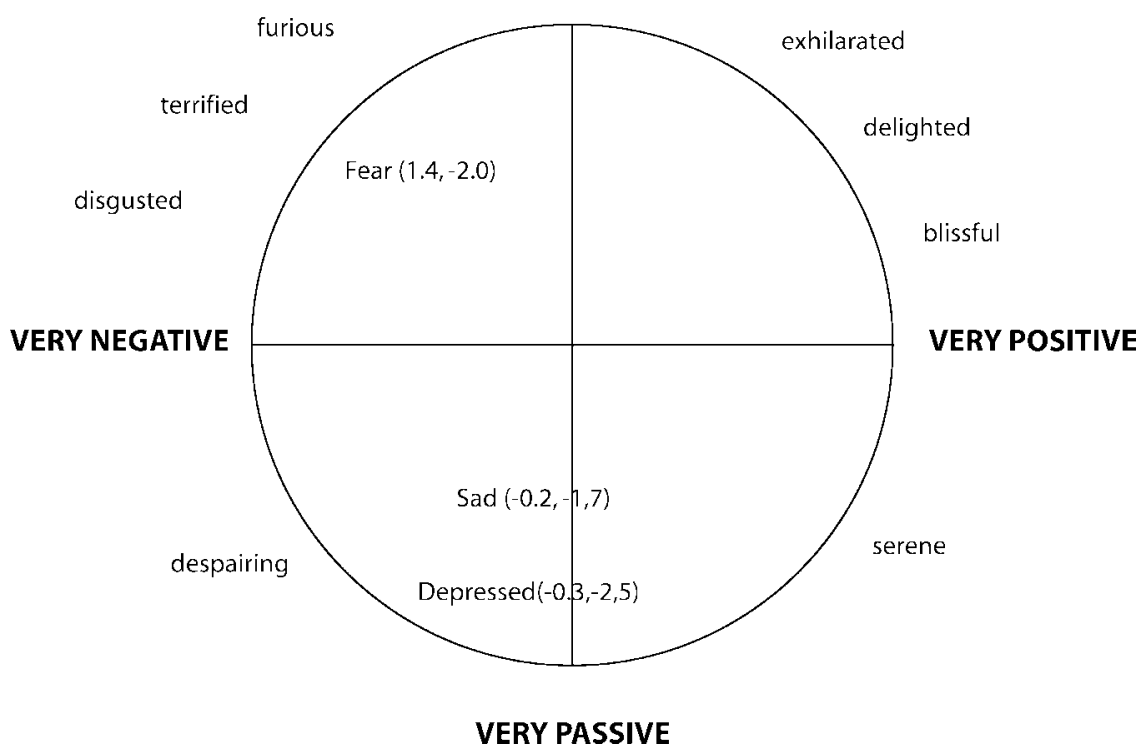

Figure 2. Activation-evaluation space.

mediate facial expressions. These profiles compound the knowledge we aim at representing in the ontology.

\section{Archetypal Expression Profile}

FAPs representations make good candidates for describing quantitative facial motion features. The use of these parameters serves several purposes such as compatibility of created synthetic sequences with the MPEG-4 standard and to increase the range of the described emotions. In general, archetypal expressions can be uniformly recognized across cultures and they are therefore invaluable in trying to analyze the user's emotional state. These expressions occur rather infrequently and in most cases emotions are expressed through a variation of a few discrete facial features related with particular FAPs.

Based on elements from psychological studies and from statistical analysis using FAPs,${ }^{1}$ we have described 25 archetypal facial expressions profiles grouped into six basic emotions. Table 1 presents the profiles created for these expressions considering ranges of FAP values for the same expression. The FAPs are represented by the $F$ and the number that it belongs to, for example, $F_{19}$ is close_t_l_eyelid. An exhaustive description of FAP can be found in MPEG-4 normative document. ${ }^{10}$ In this case, sadness has only one profile and fear has nine. Raouzaiou et al. ${ }^{1}$ present the complete list of all archetypal expression profiles and the associated FAPs ranges. For the cases where an emotion has many profiles, it means that this emotion can be expressed differently in each one of those different profiles. For example, the fear emotion profile zero describes an afraid person who has the bottom mid-lip raised, his eyes slightly closed and only the inner and middle part of his eyebrows raised; while the fourth fear profile describes a person who has his mouth and his eyes opened, his eyebrows raised and squeezed.

\section{Intermediate Expression Profile}

Creating profiles for an expression that cannot be clearly characterized as an archetypal one is not straightforward. Apart from estimating the range of variations for FAPs, one should first define which FAPs are involved in the particular expression.

One is able to synthesize intermediate expressions by combining the FAPs employed for the representation of two archetypal ones. In order to define the profiles of intermediate expressions, we used Whissel's wheel ${ }^{14}$ which suggests that expressions are points in a space. FAPs that are common in both expressions are retained during synthesis, while FAPs used in only one expression are averaged with the respective neutral position. In the case of having FAPs in common, averaging of intensities usually favors the most exaggerated of the expressions that are combined, whereas FAPs with contradicting intensities are canceled out. 


\section{computer animation

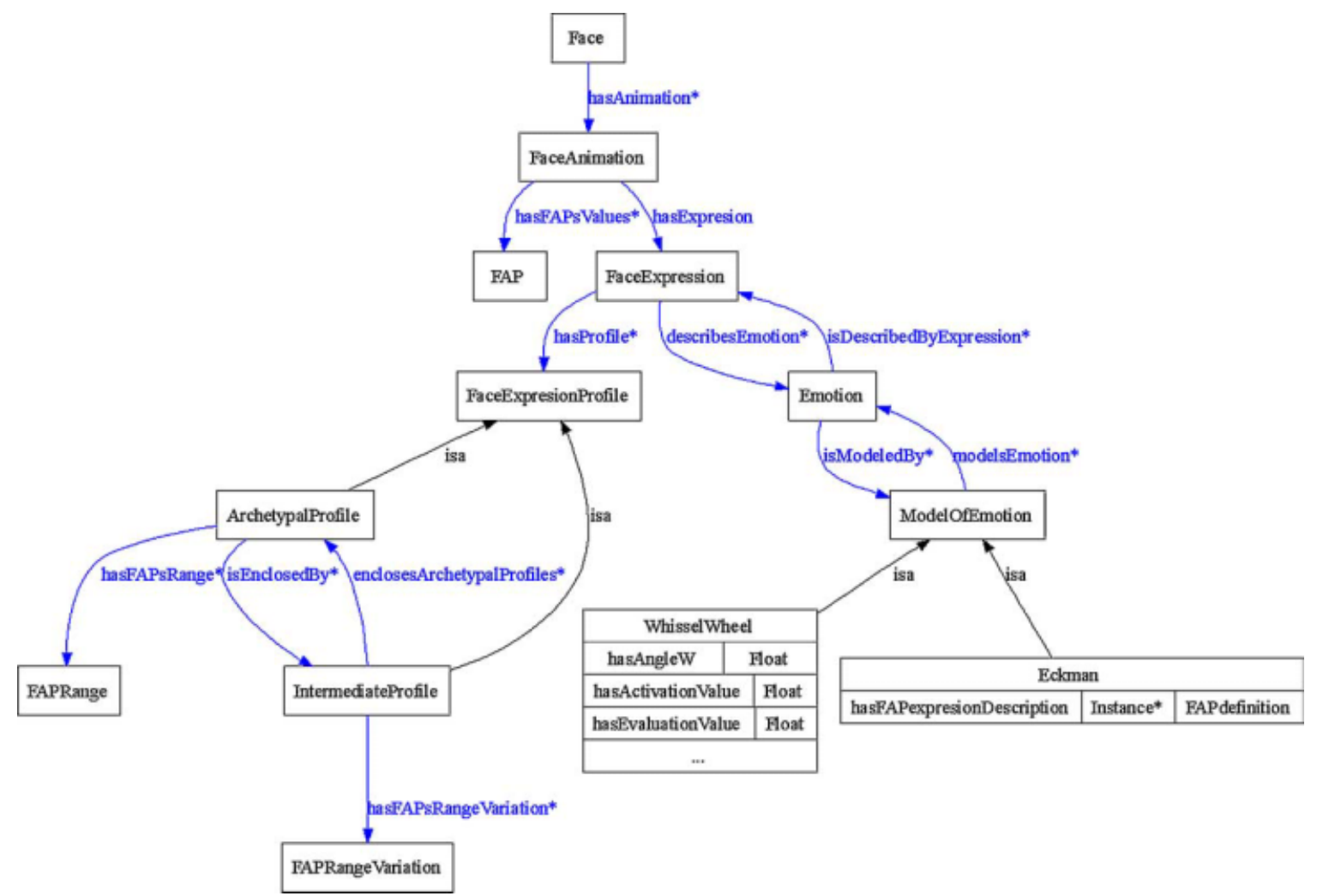

Figure 3. Description of the archetypal and intermediate face expression profiles and the representation of emotion.

This is a rule-based technique for analysis and synthesis to merge profiles of archetypal expressions for creating intermediate ones. An example of the intermediate expression depressed is presented in the Table 2 the range variation resulting of combining $\operatorname{sad}\left(P_{S}^{(0)}\right)$ and fear $\left(P_{F}^{(4)}\right)$ archetypal profiles using the activation-evaluation measures. Only some example of FAPs are presented in Table 1, Raouzaiou et al. ${ }^{1}$ present the detailed explanation and reference to intermediate expressions. The merge procedure can be better understood by an example. Let us consider a sadness profile (open eyes, inner part of eyebrows slightly raised) that is combined with a fear profile (open mouth, wide open eyes, raised eyebrows). The result of such combination is a version of the expression depression where the mouth is not as open as in fear and we have assigned to eyes and eyebrows values lying between the values of the above-mentioned profiles, so eyes are more open than in fear and less open than in sadness and the same stands for eyebrows as well.

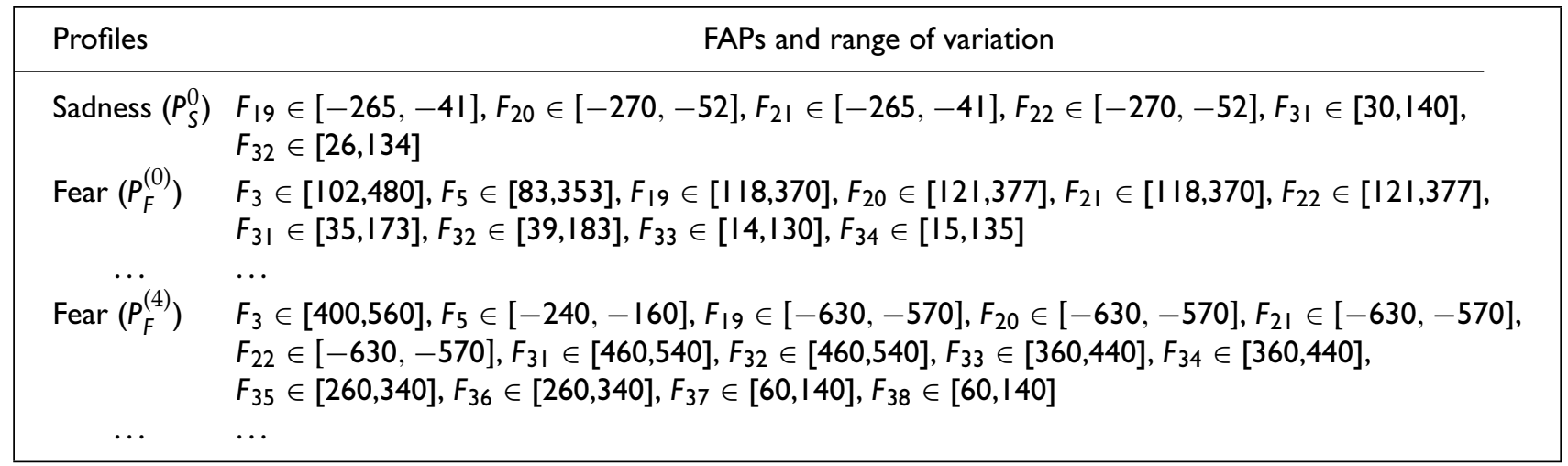

Table I. Profile for the archetypal expression sadness and Fear 


\begin{tabular}{|lcccccccc|}
\hline Expression & Activ-Eval & $F_{3} \min$ & $F_{3} \max$ & $F_{5} \min$ & $F_{5} \max$ & $F_{19 \min }$ & $F_{19 \max }$ & $\ldots$ \\
\hline Fear $\left(P_{F}^{(4)}\right)$ & $(1.4,-2.0)$ & 400 & 560 & -200 & -160 & -630 & -570 & $\ldots$ \\
Depressed $\left(P_{D}^{(0)}\right)$ & $(-0.3,-2.5)$ & 160 & 230 & -100 & -65 & -110 & -310 & $\ldots$ \\
Sad $\left(P_{S}^{(0)}\right)$ & $(-2.0,1.7)$ & 0 & 0 & 0 & 0 & -265 & -41 & $\ldots$ \\
\hline
\end{tabular}

Table 2. Intermediate profile of depression and the archetypal expressions used to calculate it using the activation and evaluation measures

\section{Ontology of Facial Expression Animation}

As already described, we have defined two types of profiles for facial expression: archetypal and intermediate. In Figure 3 we present a ontology diagram. We have defined classes and properties to describe the synthesis of emotional facial animation. In this diagram a virtual human's face class can have a facial animation that has an expression. Expressions can be described by an archetypal or intermediate profile; to describe this knowledge more efficiently, we present the next assertions defined in the ontology with the defined classes and properties:

(1) The face expression can be defined by a profile archetypal or intermediate.

(2) Each intermediate expression should enclose two archetypal expressions.

Intermediate Profile encloses Archetypal Profiles $=2$

(3) Each profile has a range of FAPs associated (FAPRange). The FAPsRange class contains the max and min FAPs values.

(4) The range of FAPs of intermediate expressions is the union of the ranges of the archetypal expression that are enclosed by the intermediate expression. This can be expressed as a restriction for the has FAPsRange property as:

$\forall$ hasFA PsRange (FA PRange $\cap((\exists$ isRangeOfExpression Profile Archetypal Profile $) \cap$ (isEnclosedBy Intermediate Profile)))

(5) Intermediate expressions FAPRangeVariation is computed considering three possibilities. (a) Mutually inclusive FAPs with different signs, (b) mutually inclusive FAPs with same sign, (c) mutually exclusive FAPs. The computation of these values is through the expression generator (this issue will be explained in the next section entitled rule population, we will also explain how the ontology could be populated and used).

\section{Ontology Rule Population}

In this section we give an example of population of expression profiles in the ontology. In Figure 4 we present a diagram of some instances for fear and sad archetypal emotional expression and depression as intermediate expression accompanied by graphical results obtained using the presented method for synthesizing face expression.

Fear emotion has a face expression FaceExpressionFear. This face expression is defined as archetypal, and as a consequence has archetypal profiles defined: ArchetypalProfiel_Fear0, ArchetypalProfiel_Fear4. Each profile has their FAPRange values obtained form. ${ }^{1}$ Sad archetypal expression (ArchetypalProfiel_Sad0) is defined in the same way.

To define depression as intermediate expression, in between of fear and sadness, we created depression face expression as an intermediate expression with its profile which encloses the archetypal expressions fear and sadness. The FAPRange of the depression profile is implicitly declared as the union of the FAP range of fear and sad profiles. For this intermediate profile the FAPRangeVariation contains the FAP range values that were obtained from the intermediate expressions generator. ${ }^{15}$ In this example we just show the FAP 19 close_t_l_eyelid which is contained in both archetypal expressions and has the same sign, which is the first case of the rules specified in the previous section.

In the same way all the possible facial expressions with their profiles should be integrated in the ontology. In the following section we will present examples of the proposed scenarios in which the ontology aims to be useful.

\section{Ontology Usage Scenarios}

The way to extract information from ontologies is by making queries. To do this we have used the plug-in 'new Racer Query Language' interface ${ }^{16}$ for OWL ontologies in Protégé. This query language is close to natural 

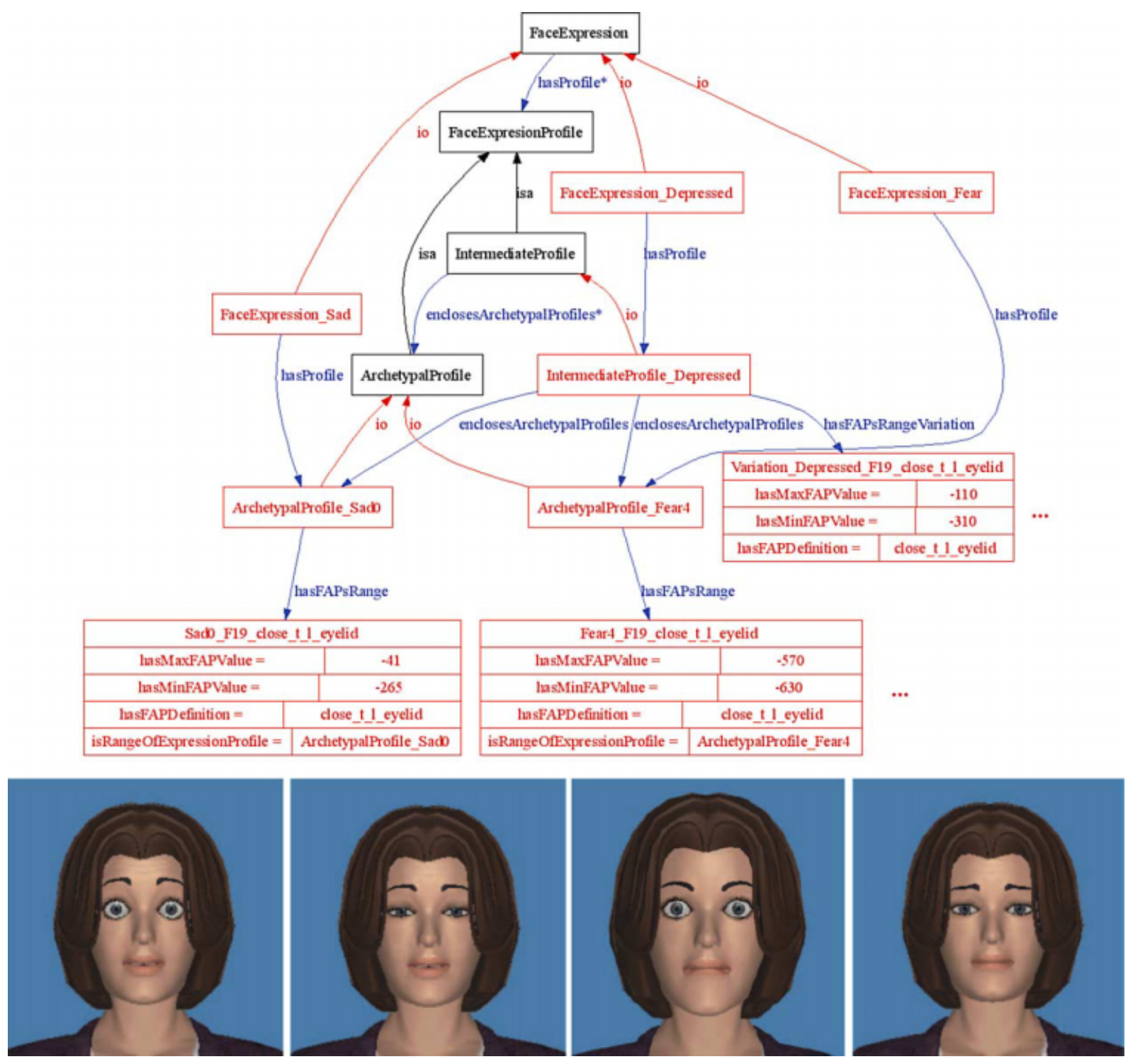

Figure 4. Example of face expression profile population and the graphical results for 1 -fear, 2 and 3-depress and 4-sadness expressions.

language. nRQL language is provided by RacerPro ${ }^{17}$ which is a system for managing semantic web ontologies based on OWL. RacerPro is necessary because it provides reasoning when querying the ontology using nRQL.

Now we present in RQL the questions that were formulated in the section entitled ontology domain. The first question belongs to the scenario where we can obtain animations that have been annotated with the structure proposed in the ontology. We present the responses to these questions we are able to obtain by populating the ontology.
In the first scenario we want to find animations that express a specific emotion.

What is the facial animation for expressing depressed emotion?

nRQL:

(retrieve (?a) (and (?a|FaceAnimation $\mid$ )

(?a $? b \mid$ hasExpresion $\mid)(? b \mid$ FaceExpression $\mid)$

$(? b$ ? $\mid$ describesEmotion $\mid)(c ? \mid$ Depressed $\mid)))$

Result: (((?A|FaceAnimation_Depressed $\mid))$

We have found that there is the FaceAnimationDepressed defined inside the ontology. In the case of the 


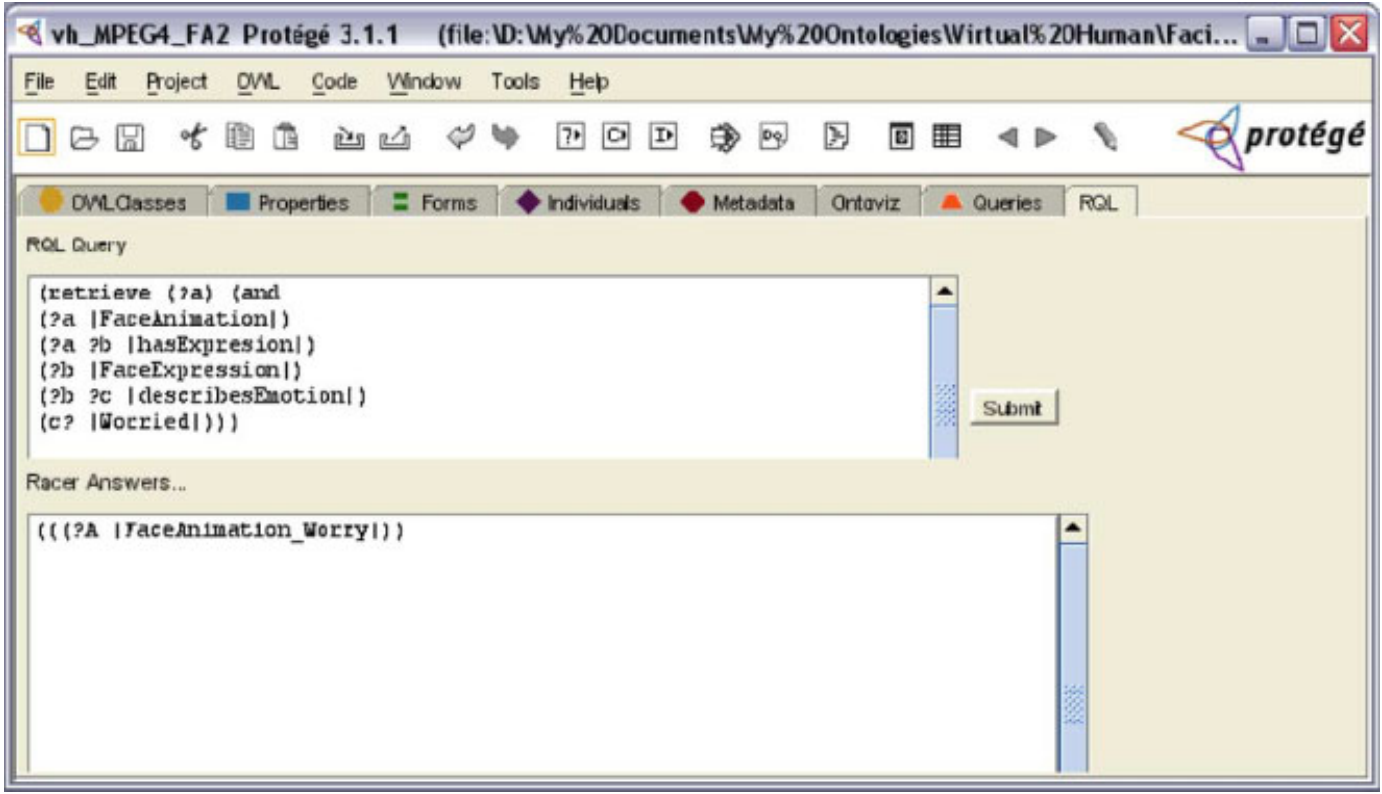

Figure 5. Snapshot of Protégé using nRQL plug-in to make queries.

second scenario probably we want to know the information used for defining this face expression, like: is the expression is intermediate or archetypal?; Which archetypal expressions it encloses? What is its FAP range? Etc.

What is the range of FAP values for the face expression depressed?

\section{nRQL:}

(retrieve (?H) (and (?A |FaceAnimation $\mid$ )

(or (and (?A ?B |hasExpresion $\mid)(? B \mid$ FaceExpression $\mid)$

(?B (some |describesEmotion $\mid$ CONCEPT - FOR Depressed $\mid))$

(?B ?F |has Profile $\mid)(? F \mid$ Intermediate Profile $\mid)$

(?F ?G|encloses Archetypal Profiles $\mid)(? G \mid$ Archetypal Profile $\mid)$

(?G ?H|hasFA PsRange $)(? H|F A P R a n g e|)))))$

Result: $\left(\left(\left(? H \mid\right.\right.\right.$ Sad $\left.\left._{F} 19 \mid\right)\right)\left(\left(? H \mid\right.\right.$ Fear $\left.\left.\left._{F} 3 \mid\right)\right)\right)$...

The last query brings the union of the set of FAPs of the archetypal expressions that are used for the intermediate depressed profile. In Figure 5 we show a screen shot of the nRQL plug-in inside Protégé when submitting a query.

\section{Conclusions and Future Work}

In this paper we have presented an ontology that demonstrates the knowledge of previous work on facial anima- tion expression within MPEG-4 framework. The facial expressions have been represented in a form of archetypal and intermediate profiles that describe the synthesis of the emotion. The structure of this ontology allows us to retrieve animations that have been annotated under this structure; and also to retrieve the expertise covered in the synthesis of those facial expressions. The main issue that is posed when adding semantics to a knowledge domain is the extraction of the meta-data and the interconnection with existing knowledge sources, such as audiovisual databases with expressive material. This issue needs to be solved as part of our future work.

Another issue that is currently being investigated is the integration of the ontology within an MPEG-4 compliant animation systems such as Greta. ${ }^{11}$ In this scenario, the animation would query the ontology for the representation of an avatar with specific expressivity, for example, a woman showing extreme joy. The result of this query would be the definition of the avatar geometry and a set of facial expressions and body gestures, ready to use in an animation. A portion of this work, is the extension of these concepts in gesturing; neutral gestures used in interaction can be transformed into expressive ones by taking into account measurable transformations, such as Laban parameters, while deictic gestures such as shrugging can vividly illustrate emotions without any further processing. Moreover, the technology available for pro- 


\section{computer animation \& virtual worlds

cessing OWL files is more focused for web as the semantic web; an on-line framework for real-time animation is still matter of research. Therefore, what we can offer under this conditions is a specific search scenarios for facial expressive animations.

\section{ACKNOWLEDGEMENTS}

This research has been funded by the EC and the Swiss Federal Office for Education and Science in the framework of the European Networks of Excellence IST-HUMAINE (http://emotionresearch.net) and IST-AIM@SHAPE (http://www.aimatshape.net).

\section{References}

1. Raouzaiou A, Tsapatsoulis N, Karpouzis K, Kollias S. Parameterized facial expression synthesis based on MPEG-4 Eurasip Journal on Applied Signal Processing 2002; 2002(10): 1021-1038.

2. Cowie R, Douglas-Cowie E, Tsapatsoulis N, et al. Emotion recognition in human-computer interaction. In IEEE Signal Processing Magazine, no. 1, January 2001, pp. 32-80.

3. Gruber TR. The role of a common ontology in achieving sharable, reusable knowledge bases. In Proceedings of the Second International Conference on Principles of Knowledge Representation and Reasoning, 1991; pp. 601-602.

4. Zaharia T, Preteux F, Preda M. 3D Shape spectrum descriptor, ISO/IEC JTC1/SC29/WG11, MPEG7/M5242, Melbourne, Australia, October 1999.

5. Zaharia T, Preteux F. Three-dimensional shape-based retrieval within the MPEG-7 framework. In SPIE Conference 4304 on Nonlinear Image Processing and Pattern Analysis XII, San Jose, CA, January 2001; pp. 133-145.

6. Gutiérrez M, Thalmann D, Vexo F, et al. An ontology of virtual humans: incorporating semantics into human shapes. In Proceedings of Workshop towards Semantic Virtual Environments (SVE05), March 2005, pp. 57-67.

7. W3C. Owl web ontology language. http://www.w3.org/ tr/owl-features/.

8. Protégé. (c) 2005 stanford medical informatics. http:// protege.stanford.edu/index.html.

9. Tekalp AM, Ostermann J. Face and 2-D mesh animation in MPEG-4. Signal Processing: Image Communication 2000; 15 $387-421$

10. ISO/IEC JTC1/SC29/WG11 N4668. Overview of the MPEG-4 standard, March 2002.

11. de Rosis F, Pelachaud C, Poggi I, Carofiglio V, De Carolis B. From greta's mind to her face: modelling the dynamics of affective states in a conversational embodied agent. International journal of Human Computer Studies 2003; 59(1-2): 81-118.

12. Koray Balci. Xface: Open source toolkit for creating $3 \mathrm{~d}$ faces of an embodied conversational agent. In Smart Graphics, pp. 263-266, 2005.

13. Eckman P. Facial expression and emotion. American Psychologist 1993; 48: 384-392.
14. Whissel CM. The dictionary of affect in language. In Emotion: Theory, Research and Experience Vol. 4, The Measurement of Emotions, Plutchnik R, Kellerman H (eds). Academic Press: New York, 1989

15. Raouzaiou A, Spyrou E, Karpouzis K, Kollias S. Emotion synthesis: an intermediate expressions generator system in the MPEG-4 framework. International Workshop VLBV05, 15-16 September 2005, Sardinia, Italy, 2005.

16. nRQL. new racer query language (nrql) interface to owl ontologies in protégé. http:/ / www.cs.concordia.ca/^ kbhoopa/nrql.html.

17. RacerPro. Renamed abox and concept expression reasoner. http://www.racersystems.com.

\section{Authors' biographies:}

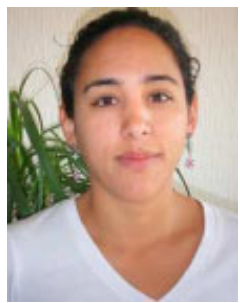

Alejandra García-Rojas graduated from Instituto Tecnológico y de Estudios Superiores de Monterrey as Computer Scientist. She received her M.T.D.I degree from the University Complutense of Madrid in 2004. Since January of 2005 she is Ph.D. student in Virtual Reality Lab (VRlab) at the École Polytechnique Fédérale de Lausanne.

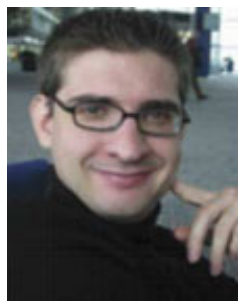

Dr Frederic Vexo is currently Nodia senior researcher and project leader at the Virtual Reality Laboratory at the École Polytechnique Fédérale de Lausanne., Switzerland. His research interests are multidisciplinary and include Human to Inhabited Virtual World, Haptic Interfaces, Tele Operated System, Multimodal Adaptive Interface for virtual worlds, Semantic Virtual Environment and new services for graphic mobile devices. He has authored several papers in journals and international conferences in the fields of Human Computer Interaction, Robotics and Computer Graphics. He is a member of several conference program committees (AAMAS, SMI, SVE and IWVR) and expert for different institution and companies. He received his Ph.D. in Computer Science in 2000 from University of Reims and Bachelor's and Master's degrees from University of METZ. He also has contributed to various European Projects. 


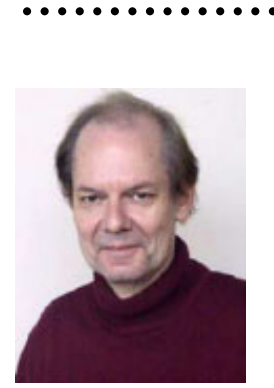

Dr Daniel Thalmann is Professor and Director of The Virtual Reality Lab (VRlab) at EPFL, Switzerland. He is a pioneer in research on Virtual Humans. His current research interests include Real-time Virtual Humans in Virtual Reality, Networked Virtual Environments, Artificial Life, and Multimedia. Daniel Thalmann has been Professor at The University of Montreal and Visiting Professor/Researcher at CERN, University of Nebraska, University of Tokyo, and Institute of System Science in Singapore. He is coeditor-in-chief of the Journal of Visualization and Computer Animation, and member of the editorial board of the Visual Computer and three other journals. Daniel Thalmann was member of numerous Program Committees, Program Chair of several conferences and chair of the Computer Graphics International 1993, Pacific Graphics 1995, ACM VRST 1997, and MMM 1998 conferences. He was Program Cochair of IEEE VR 2000. He has also organized four courses at SIGGRAPH on human animation. Daniel Thalmann is member of Eurographics since 1980. He was the initiator of the Eurographics working group on Animation and Simulation which he co-chaired during more than 10 years. He is now member of the Stearing Committee of the Symposium on Computer Animation, a joint Eurographics-Siggraph initiative. Daniel Thalmann has published more than 300 papers in Graphics, Animation, and Virtual Reality. He is coeditor of 30 books, and coauthor of several books including the 'Handbook on Virtual Humans', published by John Wiley and Sons. He was also co-director of several computer-generated films with synthetic actors including a synthetic Marilyn shown on numerous TV channels all over the world. He received his Ph.D. in Computer Science in 1977 from the University of Geneva and an Honorary Doctorate (Honoris Causa) from University Paul-Sabatier in Toulouse, France, in 2003.

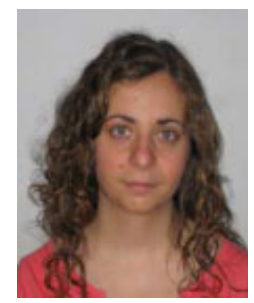

Amaryllis Raouzaiou graduated from the Department of Electrical and Computer Engineering, the National Technical University of Athens in 2000. She is currently pursuing her Ph.D. degree at the Image, Video, and Multimedia Systems Laboratory at the same University. Her current research interests lie in the areas of Human-computer interaction, Synthetic-natural Hybrid Video Coding and Machine Vision. She is a member of the Technical Chamber of Greece. She is with the team of NoE HUMAINE (IST-2002-2.3.1.6 Multimodal Interfaces.).

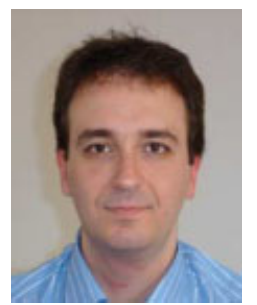

Dr. Kostas Karpouzis graduated from the Department of Electrical and Computer Engineering, of the National Technical University of Athens in 1998 and received his Ph.D. degree in 2001 from the same University. His current research interests lie in the areas of human computer interaction, image and video processing, 3D computer animation, sign language synthesis and virtual reality. Dr. Karpouzis has published more than 70 papers in international journals and proceedings of international conferences. He is a member of the technical committee of the International Conference on Image Processing (ICIP) and a reviewer in many international journals. Since 1995 he has participated in more than 10 research projects at Greek and European level. Dr. Karpouzis is an associate researcher at the Institute of Communication and Computer Systems (ICCS) and holds an adjunct lecturer position at the University of Piraeus, teaching Medical Informatics and Image Processing. He is also a national representative in IFIP Working Groups 12.5 'Artificial Intelligence Applications' and 3.2 'Informatics and ICT in Higher Education'.

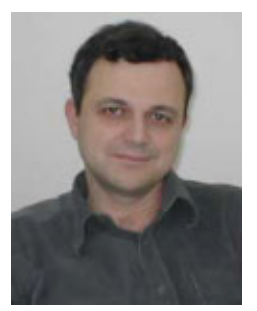

Prof. Stefanos Kollias was born in Athens in 1956. He obtained his Diploma from NTUA in 1979, his M.Sc. in Communication Engineering in 1980 from UMIST in England and his Ph.D. in Signal Processing from the Computer Science Division of NTUA. He is with the Electrical Engineering Department of NTUA since 1986 where he serves now as a Professor. Since 1990 he is Director of the Image, Video and Multimedia Systems Laboratory of NTUA. He has published more than 120 papers in the above fields, 50 of which in international journals. He has been a member of the Technical or Advisory Committee or invited speaker in 40 International Conferences. $\mathrm{He}$ is a reviewer of 10 IEEE Transactions and of 10 other journals. Fifteen graduate students have completed their 
Doctorate under his supervision, while another fifteen are currently performing their Ph.D. thesis. He and his team have been participating in more than 40 European and National projects. Prof. Kollias is also a member of the NRG Group representing Greece in matters of digitization and preservation of audiovisual material.

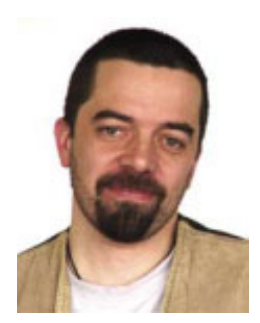

Laurent Moccozet is a senior researcher at MIRALab, University of Geneva. His main research field is geometric modeling and geometric deformations for articulated characters such as virtual humans. He is also investigating semantics-based representation for high-level models of virtual humans. He has been actively involved in several European and Swiss Projects focused on human shape modeling for virtual environments.

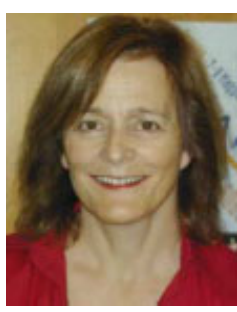

Nadia Magnenat-Thalmann has pioneered research in virtual humans over the last 20 years. She studied at University of Geneva and obtained several degrees in Psychology, Biology, Chemistry, and a Ph.D. in quantum mechanics from University of Geneva in 1977. From 1977 to 1989 , she was a professor at University of Montreal, Canada. In 1989, she founded MIRALab, an interdisciplinary creative research laboratory at University of Geneva. She has published more than 200 papers, and is editor-in-chief of Visual Computer and the Journal of Visualization and Computer Animation journals. She has received several awards, and recently she has been nominated at the Swiss Academy of Technical Sciences. 